\title{
Application for UROgEnital Disorders
}

\section{Yoshikatsu TANAHASHI, M.D.}

Department of Urology, Tohoku Univ. School of Med., Sendai,Japan

Three kinds of lasers, namely carbon dioxide laser, neodymium YAG laser and argon laser are used today for laser treatment in the field of urology. Neodimium YAG laser and argon laser are suitable for the endoscopic use in urology than carbon dioxide laser, because these two kind of lasers are guided through fine optical fibers, so no special equipment is necessary for endoscopic laser surgery when it is done using these two kind of lasers.

The actual procedures of the treatment of caruncle, penile cancer, bladder tumor, urethral stenosis and of urinary stones are shown in this lecture.

URETHRAL CARUNCLE

Treatment of urethral caruncle using laser beam is similar to the electrocautery. Unfocused or defocused beam of YAG laser or $\mathrm{CO}_{2}$ laser is irradiated to the tumor causing vaporization.

\section{PENILE CANCER}

Amputation of the penis in the cases of penile cancer is one of the most suitable application of the laser beam. Because penile shaft is composed of bloody structures called corpora cavernosa and corpus spongiosum. Amputation is performed with focused beam of YAG laser and cut end of the penis is coagulated with defocused beam. During these procedures, no bleeding is observed.

\section{BLADDER TUMOR}

Treatment of bladder tumor using laser beam is performed in our clinic in combination with transurethral electroresection(TUR). At first, hot biopsy of the tumor is made and rough resection of the tumor is done. And then laser should be irradiated for hemostasis and

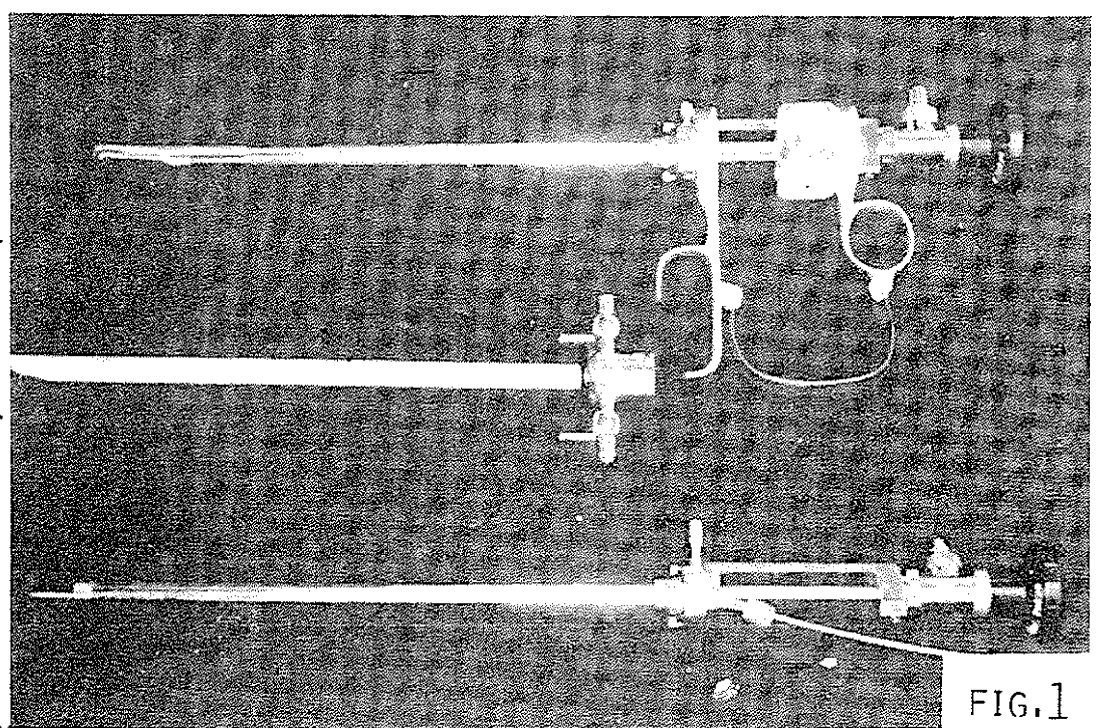


additional resection and cleaning up of the resected floor.

is troublesome to change resectoscope for TUR and cystoscope for laser irradiation in and out of the urethral tract. The author made an adaptor, as shown in Fig.l, so that the outer jaket sheath may be installed into the urethral tract and into the bladder. And thus the loop for TUR and optical fiber for laser may be changed by one step with ease.

URETHRAL STRICTURE

Ureteral catheter is inserted via the catheter passage and under its guidance YAG laser beam is irradiated at 12 O'clock portion. Once the stenotic portion is dissected, the endoscope may be advanced further for irradiation of the more proximal portion.

URINARY CALCULI

Destruction of the vesical stone is performed as in the case of cystoscopy. After insertion of the cystoscope, bladder is filled with 150-200ml of water. Then the optical fiber is introduced into the bladder from the catheter passage. YAG laser beam is irradiated to the stone under direct vision. As the stone is destructed, fine tips of the stone make the intravesical fluid turbid, and then the temperture of the fluid is gradually elevated by the thermal effect of the laser. So the fluid should be changed as in the case of cystoscopic examination procedure. When the stone is destructed into pieces of several millimeter in size, they are removed out of the bladder by means of forceps or evacuator.

Destruction of renal or ureteral stone is performed through nephrostomy channel made percutaneously. Renal pelvic puncture using real time ultrasonic guidance is performed at first. Supported by a guide wire, the punctured hole is enlarged by means of telescopic metal dilator finally up to $8 \mathrm{~mm}$ in diameter. Then a $24 \mathrm{~F}$ balloon catheter is inserted. Destruction of the stone is performed about 3 to 5 days after the installation of the renal fistula. Rigid nephroscope which has large capacity to send large amount of cooling water is introduced into the renal pelvis via the way of nephrostomy channel. And YAG laser beam is irradiated to the stone under direct vision. Once the stone was tend to be destructed, it is very effective to add small out-force to the stone by means of forceps or ultrasonic vibration. When the pieces of the stone became so small that they could pass through the nephrostomy channel, a forceps might be most suitable tool to take out the cracked stone. 